\title{
Análisis histórico de la efectividad de los lanzamientos a portería de balonmano masculino Historical analysis of throwing effectiveness in male handball
}

\author{
Jose Manuel Jimenez-Olmedo, Jose Julio Espina-Agullo and Carmen Manchado
}

Universidad de Alicante (España)

\begin{abstract}
Resumen. El objeto de este artículo es realizar un análisis de la efectividad de los lanzamientos en el alto rendimiento en balonmano. Para ello la muestra recoge 32 finales de Campeonatos del Mundo, de Europa y Juegos Olímpicos desde 1982 hasta 2012. El análisis de los lanzamientos se ha efectuado a partir de diferentes zonas, estableciendo como referencia la línea de 6 metros (centro y lateral), 7 metros y 9 metros (centro y lateral), e incluyendo los lanzamientos en situación de contraataque. El análisis de las videograbaciones se llevó a cabo con el software SportCode Pro v.8.5.2. La comparación de las frecuencias de las diferentes zonas de lanzamiento analizadas, se empleó la prueba Kruskal-Wallis. Los resultados demuestran las diferentes evoluciones de lanzamiento para cada una de las zonas analizadas. Se destaca el aumento de los lanzamientos de zona de 9 metros centro $(9 \mathrm{mC})(p=0,014)$ a lo largo de los periodos analizados. En cuanto a la evolución de goles, se destaca el aumento de los goles conseguidos en zona de 6 metros lateral, siendo mayor que en 6 metros centro $(6 \mathrm{mC})$. Finalmente se establece la evolución de la efectividad para cada una de las zonas analizadas, destacando el aumento de la efectividad para los lanzamientos de 7 metros (del 52,6\% al 76,7\%) y contraataque (del 55,6\% al 71,1\%).

Palabras clave: Análisis notacional, balonmano, efectividad de lanzamiento, lanzamiento.
\end{abstract}

Abstract. The aim of this paper is to analyze the throwing effectiveness in high performance handball. Therefore, the sample comprises 32 World Championships, European Championships and Olympic Games finals from 1982 to 2012. The analysis of throwing has been conducted from different areas, establishing as reference the line of 6 meters (center and lateral), 7 meters and 9 meters (center and lateral), and including the throwing in counterattack situation. Video recordings were analyzed using SportCode Pro v.8.5.2 software and the Kruskal-Wallis test was used to compare the different throwing areas frequencies. As for the results, they show the different throwing evolutions for each of the analyzed area. It highlights the increase of 9 meters center throws $(9 \mathrm{mC})(\mathrm{p}=0.014)$ during the analyzed periods. Concerning the number of goals, it is also emphasized that the goals scored from 6 meters lateral $(6 \mathrm{~mL})$ were larger than from 6 meters center $(6 \mathrm{mC})$ Finally, it is set the evolution of effectiveness for each one of the analyzed areas, with an increase in effectiveness for the 7 meters throw (from 52.6\% to 76.7\%) and counterattack throwing (from 55.6\% to $71.1 \%$ ). Key words: Notational analysis, handball, throw efectivity, throw.

\section{Introducción}

El lanzamiento de balonmano ha sido estudiado en profundidad, desde categorías amateur a profesionales (García, Moreno, Reina, \& Menayo, 2011; Granados, Izquierdo, Ibañez, Bonnabau, \& Gorostiaga, 2007), para poder conocer, de este modo, los factores condicionales que determinan su calidad, precisión y velocidad.

Para poder contar con una buena calidad de lanzamiento se deben tener en cuenta aspectos psicológicos (Peñaloza, Jaenes, MendezSanchez y Haenes-Amarillo, 2016; González y Valadez, 2016), métodos de entrenamiento y mejora técnica (Pascual, Alzamora, Martinez y Perez, 2015) así como un control de la carga de entrenamiento y los periodos de descanso (Reynoso-Sanchez et al., 2016; Gonzalez-Fimbres, Griego, Cuevas-Castro y Hernandez, 2016; Murillo, Alvarez y Manomelles, 2016). Algunos de estos elementos son entrenables, pudiendo obtener una mejora en el rendimiento y por tanto, de la efectividad del lanzamiento a través de programas específicos de entrenamiento basados en la mejora de la fuerza, la especificidad y la velocidad (Van den Tillaar, 2004).

Del mismo modo, se debe tener en cuenta que la salida de balón depende de un extenso conjunto de elementos, especialmente relacionados con la técnica de ejecución y las características antropométricas y físicas de los lanzadores. Por lo que respecta a la técnica de ejecución, son diversos los estudios que se han centrado en el análisis del lanzamiento y su relación con la precisión y velocidad del balón (Van den Tillaar, 2003; Zapartidis, Gouvali, Bayios, \& Boudolos, 2007) estableciéndose una correlación donde la velocidad afecta negativamente a la precisión del lanzamiento, por lo que los autores recomiendan el trabajo tanto de precisión como de velocidad específico para la mejora de estos aspectos en los lanzadores. Además, se han estudiado los lanzamientos tanto con brazo dominante como no dominante (Van den Tillaar \& Ettema, 2009), modificando las posiciones del brazo en la ejecución (Wagner, Buchecker, Von Duvillard, \& Müller, 2010) así como el uso de diferentes técnicas de lanzamiento (Wagner, Pfusterschmied, Klous, von Duvillard, \& Müller, 2012) donde se analizan en todos ellos, la relación de la velocidad y la precisión. Llegando incluso a analizar los lanzamientos de forma específica en el salto, siendo una técnica ampliamente empleada en el balonmano (Wagner, Buchecker, von Duvillard, \& Müller, 2010). De este modo se establece una relación directa entre la precisión y la velocidad del lanzamiento relacionada con la velocidad de extensión del codo y la rotación interna del hombro (Van den Tillaar \& Ettema, 2004a)

Por otro lado, en cuanto a las características de los lanzadores, se ha estudiado en profundidad el perfil físico y antropométrico en relación al lanzamiento (Vila et al., 2012), estableciéndose los diferentes perfiles de los jugadores por posición de juego (Rogulj, Srhoj, Nazor, Srhoj, \& Cavala, 2005). Se establece así mismo una relación entre la masa corporal y la velocidad de lanzamiento (Van den Tillaar \& Ettema, 2004b), siendo las características antropométricas y la media de $1 \mathrm{RM}$ en press banca, predictores de la velocidad de lanzamiento (Debanne \& Laffaye, 2011; Marques, van den Tilaar, Vescovi, \& Gonzalez-Badillo, 2007).

Siguiendo con el análisis de los factores condiciones del lanzamiento, no todos dependen del lanzador. De todos ellos se destaca la oposición a la que se enfrenta (Rivilla-Garcia, Grande, Sampedro, \& van den Tillaar, 2011), el papel del portero (Espina-Agulló, Pérez-Turpin, Jiménez-Olmedo, Penichet-Tomás, \& Pueo, 2016), así como las características antropométricas de la defensa. Todos estos factores externos al lanzador, condicionan su respuesta de ataque y adaptación de los patrones técnicos para sobrepasar las dificultades encontradas para poder realizar un lanzamiento de calidad que permita la obtención del tanto.

Los factores anteriormente expuestos son los que condicionan la calidad del lanzamiento y por consiguiente, el hecho de tener una mayor o menor efectividad, la cual se relaciona directamente con la velocidad y precisión del mismo (Manchado, Tortosa-Martínez, Vila, Ferragut, \& Platen, 2013). Pero se debe tener en cuenta, que el desarrollo del partido y la acumulación de la fatiga afecta negativamente a la efectividad del lanzamiento debido a la perdida de precisión (Zapartidis et al., 2007).

Todo lo anterior explica desde un punto de vista técnico los factores condicionales de lanzamientos y los elementos o situaciones de juego que afectan a su ejecución y por tanto a la consecución o no del tanto.

Siguiendo esta línea, es importante conocer las efectividades de lanzamiento por cada una de las zonas de juego posibles, lo cual ayuda a establecer estrategias de juego y a comprender mejor la dinámica del 
mismo. En este punto se establece la necesidad de conocer y emplear metodologías adecuadas que a través del análisis, nos aporten información relevante sobre este aspecto. Pero a pesar de ello, se debe tener en cuenta que la mejora de los jugadores, un mayor conocimiento del deporte y de los métodos de entrenamiento, sugieren que junto a las modificaciones del reglamento y la evolución de los sistemas de juego (Espina-Agullo, 2013; Espina-Agulló \& Jove-Tossi, 2012), se haya producido una evolución en cuanto a las efectividades de los lanzadores con el paso del tiempo.

Dicho esto, se plantea la necesidad de conocer y estudiar la evolución de los lanzamientos y más concretamente de las efectividades de lanzamiento con el paso del tiempo. Por tanto, el objetivo de este estudio es realizar un análisis de las efectividades de lanzamiento de 1982 a 2012

\section{Método}

\section{Muestra}

La muestra se centra en el estudio de las finales pertenecientes a Campeonatos del Mundo, Campeonatos de Europa y Juegos Olímpicos de categoría masculina, comprendidas entre los años 1982 y 2012. Por tanto la muestra cuenta con 10 finales europeas, 14 finales de campeonato del mundo y 8 finales olímpicas.

El material de videograbación sobre el que se ha llevado a cabo el análisis, se obtuvo de las retransmisiones realizadas en TV1, TV2, TDP y Eurosport. Para llevar a cabo el análisis de las videograbaciones, se ha utilizado el software SportCode Pro v. 8.5.2.

En total 3690 acciones en las que se analizan todos los lanzamientos realizados durante los encuentros anteriormente citados.

\section{Procedimientoyvariables}

La visualización y análisis de las videograbaciones se llevó a cabo por 2 observadores experimentados (Jimenez-Olmedo, Pueo, \& Penichet-Tomás, 2016). Para la realización de la fiabilidad de la observación durante el estudio, se llevaron a cabo 2 visualizaciones inter-operador (Jimenez-Olmedo, Pueo, Penichet-Tomás, Chinchilla-Mira, \& Perez-Turpin, 2017). Además, para cada una de las variables utilizadas se llevó a cabo un cálculo del error de la observación siguiendo la siguiente expresión (Hughes, 2004)

$\operatorname{Diff}(\%)=(\operatorname{Imod}(V 1-V 2)) /($ Vmean $* 100)$

donde V1 y V2 son las frecuencias observadas tanto por el observador 1 como por el observador 2 variables, Vmean es el valor medio de las frecuencias de las dos observaciones realizadas y mod es el módulo.

\begin{tabular}{|c|c|}
\hline \multicolumn{2}{|c|}{$\begin{array}{l}\text { Matriz de Análisis zona de lanzamientos } \\
\text { Operción Definción }\end{array}$} \\
\hline & $\begin{array}{l}\text { Definición } \\
\text { Lanzamientos realizados en la zona comprendida entre la línea de } 6 \text { metros y la de }\end{array}$ \\
\hline $6 \mathrm{mC}$ & $\begin{array}{l}9 \text { metros. En los lanzamientos donde el jugador se encuentra en el aire, se tomará } \\
\text { como zona de lanzamiento el lugar desde el cual realizó la batida previa al vuelo. } \\
\text { Además se contemplan solo los lanzamientos realizados desde la zona central del } \\
\text { área. }\end{array}$ \\
\hline $6 \mathrm{~mL}$ & $\begin{array}{l}\text { Lanzamientos realizados en la zona comprendida entre la línea de } 6 \text { metros y la de } \\
9 \text { metros. En los lanzamientos donde el jugador se encuentra en el aire, se tomará } \\
\text { como zona de lanzamiento el lugar desde el cual realizó la batida previa al vuelo. } \\
\text { Además se contemplan solo los lanzamientos realizados desde la zona lateral del } \\
\text { área, ya sean por izquierda o por derecha. }\end{array}$ \\
\hline $9 \mathrm{mC}$ & $\begin{array}{l}\text { Lanzamientos realizados por detrás la línea de } 9 \text { metros delimitada en el campo de } \\
\text { juego. En los lanzamientos donde el jugador se encuentra en el aire, se tomará } \\
\text { como zona de lanzamiento el lugar desde el cual realizó la batida previa al vuelo. } \\
\text { Además se contemplan solo los lanzamientos realizados desde la zona central del } \\
\text { área. }\end{array}$ \\
\hline $9 \mathrm{~mL}$ & $\begin{array}{l}\text { Lanzamientos realizados por detrás la línea de } 9 \text { metros delimitada en el campo de } \\
\text { juego. En los lanzamientos donde el jugador se encuentra en el aire, se tomará } \\
\text { como zona de lanzamiento el lugar desde el cual realizó la batida previa al vuelo. } \\
\text { Además se contemplan solo los lanzamientos realizados desde la zona lateral del } \\
\text { área, ya sean por izquierda o por derecha. }\end{array}$ \\
\hline $7 \mathrm{~m}$ & $\begin{array}{l}\text { Se toma como lanzamiento de } 7 \text { metros todos aquellos que se realicen desde dicha } \\
\text { distancia por indicación del árbitro. }\end{array}$ \\
\hline Contra & Lanzamientos realizados en situación de contraataque. \\
\hline \multicolumn{2}{|c|}{$\begin{array}{l}\text { Nota: 6mC: Lanzamientos de seis metros centro; 6mL: Lanzamientos de seis metros lateral; } \\
\text { 9mC: Lanzamientos de nueve metros centro; 9mL: Lanzamientos de nueve metros lateral; 7m: } \\
\text { Lanzamientos de siete metros; Contra: Lanzamiento después de contraataque. }\end{array}$} \\
\hline \multicolumn{2}{|c|}{$\begin{array}{l}\text { Tabla 2: } \\
\text { Matriz de Análisis lugares de lanzamientos }\end{array}$} \\
\hline Operación & Definición \\
\hline Gol & $\begin{array}{l}\text { Aquellos lanzamientos en los que el portero no detiene el balón y este acaba } \\
\text { entrando en la portería. }\end{array}$ \\
\hline No Gol & $\begin{array}{l}\text { Aquellos lanzamientos en los que por intervención del portero o error del lanzador, } \\
\text { el balón no entra en la portería. }\end{array}$ \\
\hline
\end{tabular}

La fiabilidad realizada sobre el análisis inter-observadores obtuvo un margen de error inferior al 5\% (James, Taylor, \& Stanley, 2007).

El análisis de los partidos analizados se centró en los lanzamientos de los atacantes desde diferentes zonas. Para ello se establecieron las variables de observación para llevar a cabo el análisis notacional de las acciones desarrolladas que ayuden a determinar la efectividad del ataque. Se establecieron 6 zonas de ataque: lanzamientos desde 6 metros centro (6mC), 6 metros lateral (6mL), 9 metros centro (9mC), 9 metros lateral $(9 \mathrm{~mL}) 7$ metros $(7 \mathrm{~m})$ y contraataque (Contra). Teniendo en cuenta las zonas de lanzamiento, se estableció el siguiente protocolo de observación(Tabla 1).

Por otro lado, para poder establecer y calcular la efectividad de los lanzamientos realizados, se determinaron las variables correspondientes al resultado de ese lanzamiento, siendo gol (GOL) o no gol (NGOL). De este modo, se establece una matriz de observación para el análisis del resultado del lanzamiento (Tabla 2).

Por tanto, para llevar a cabo el análisis de los partidos, se siguió la siguiente secuencia:

- Grabación y digitalización de las imágenes.

- Creación de matrices de código con los elementos a analizar para cada análisis.

- Codificación de las imágenes con cada uno de los códigos creados.

- Combinación de los códigos obteniendo una cuantificación de las acciones estudiadas.

\section{Análisis estadístico}

Los análisis fueron realizados con el software SPSS v.22. En primer lugar se aplicó la prueba de normalidad Kolmogorov-Smirnov, y seguidamente la prueba para la comparación de las proporciones $\mathrm{H}$ test de Kruskal-Wallis, con un intervalo de confianza del 95\%. Dicho test, evaluó la comparación de frecuencias de acciones para cada una de las zonas de lanzamiento en función del ciclo olímpico en que se realizaron.

\section{Resultados}

\section{Lanzamientos totales}

Los resultados obtenidos del análisis de los partidos, muestran en primer lugar, el número total de lanzamientos medios por cada una de las zonas de lanzamientos analizadas, con independencia de la efectividad de dicho lanzamiento. Todos y cada uno de ellos, fueron clasificados en función del ciclo olímpico en el que se produjeron (Tabla 3).

\begin{tabular}{|c|c|c|c|c|c|c|c|c|}
\hline & & $\begin{array}{l}\text { Media } \\
\text { (M) }\end{array}$ & $\begin{array}{l}\text { esviación } \\
\text { (SD) }\end{array}$ & $\begin{array}{c}\text { Mediana } \\
(\mathrm{Me})\end{array}$ & & $\begin{array}{l}\text { Media } \\
\text { (M) }\end{array}$ & $\begin{array}{l}\text { Desviación } \\
\text { (SD) }\end{array}$ & $\begin{array}{c}\text { Mediana } \\
\text { (Me) }\end{array}$ \\
\hline \multirow[t]{9}{*}{$6 \mathrm{mC}$} & \multicolumn{8}{|c|}{$9 \mathrm{mC}$} \\
\hline & Ciclo 1 & 4,75 & 3,49 & 4 & Ciclo 1 & 3,63 & 2,06 & 4,5 \\
\hline & Ciclo 2 & 4,75 & 4,4 & 3,5 & Ciclo 2 & 3,13 & 2,69 & 2,5 \\
\hline & Ciclo 3 & 5 & 2,2 & 5,5 & Ciclo 3 & 4,38 & 2,77 & 3,5 \\
\hline & Ciclo 4 & 4,6 & 2,81 & 4 & Ciclo 4 & 2,15 & 1,53 & 2 \\
\hline & Ciclo 5 & 5,15 & 2,77 & 4,5 & Ciclo 5 & 3,2 & 1,93 & 3 \\
\hline & Ciclo 6 & 5,2 & 3,76 & 5,5 & Ciclo 6 & 4,3 & 2,34 & 4,5 \\
\hline & Ciclo 7 & 6,05 & 3,33 & 5 & Ciclo 7 & 4,7 & 2,31 & 5 \\
\hline & Ciclo 8 & 4,05 & 2,32 & 3,5 & Ciclo 8 & 4,8 & 3,07 & 4,5 \\
\hline \multirow[t]{9}{*}{$6 \mathrm{~mL}$} & \multicolumn{8}{|c|}{$9 \mathrm{~mL}$} \\
\hline & Ciclo 1 & 5,75 & 3,49 & 5,5 & Ciclo 1 & 0,75 & 1,03 & 0,5 \\
\hline & Ciclo 2 & 5,75 & 2,65 & 5,5 & Ciclo 2 & 1 & 1,19 & 0,5 \\
\hline & Ciclo 3 & 5,88 & 2,99 & 6 & Ciclo 3 & 1,13 & 1,35 & 0,5 \\
\hline & Ciclo 4 & 6,25 & 2,84 & 6 & Ciclo 4 & 1,1 & 1,07 & 1 \\
\hline & Ciclo 5 & 8 & 2,71 & 7 & Ciclo 5 & 1,4 & 1,27 & 1 \\
\hline & Ciclo 6 & 6,35 & 3,08 & 7 & Ciclo 6 & 0,95 & 1,05 & 0,5 \\
\hline & Ciclo 7 & 6,15 & 2,34 & 6 & Ciclo 7 & 0,9 & 0,912 & 1 \\
\hline & Ciclo 8 & 6,8 & 3,01 & 6 & Ciclo 8 & 1 & 0,97 & 1 \\
\hline \multirow{9}{*}{ Contra } & \multicolumn{8}{|c|}{$7 \mathrm{~m}$} \\
\hline & Ciclo 1 & 2,25 & 1,58 & 2,5 & Ciclo 1 & 2,38 & 2,2 & 2 \\
\hline & Ciclo 2 & 3 & 0,92 & 3 & Ciclo 2 & 2,25 & 1,98 & 2,5 \\
\hline & Ciclo 3 & 2,88 & 1,45 & 2,5 & Ciclo 3 & 1,63 & 1,76 & 1 \\
\hline & Ciclo 4 & 3,2 & 2,37 & 3 & Ciclo 4 & 1,55 & 1,76 & 1 \\
\hline & Ciclo 5 & 2,65 & 1,72 & 3 & Ciclo 5 & 1,85 & 1,56 & 2 \\
\hline & Ciclo 6 & 2,1 & 1,58 & 2 & Ciclo 6 & 1,9 & 1,8 & 1,5 \\
\hline & Ciclo 7 & 3,2 & 2,16 & 3 & Ciclo 7 & 1,6 & 1,35 & 1 \\
\hline & Ciclo 8 & 1,9 & 1,65 & 1 & Ciclo 8 & 2,15 & 1,98 & 2 \\
\hline
\end{tabular}

Para los lanzamientos desde 6 metros, se observa una tendencia irregular en el uso del lanzamiento de 6 metros centro (6mC), la cual ha 
evolucionado muy poco en cuanto a la frecuencia de uso, pues son acciones de ataque con finalización por parte del pivote, siendo esta una opción recurrente en lanzamientos por esta zona. Por el contrario, el lanzamiento de 6 metros lateral $(6 \mathrm{~mL})$, si ha experimentado una evolución positiva, realizándose un mayor número de lanzamientos desde estas zonas de ataque a lo largo del tiempo, lo que sugiere una mejora técnica de los lanzadores, que han resuelto la falta de verticalidad, con lanzamientos de rosca o liftados que no eran utilizados hace 30 años.

A pesar de ello, no se establecen diferencias estadísticamente significativas para lanzamientos de 6 metros centro $(6 \mathrm{mC})(p=0,523)$ y 6 metros lateral $(6 \mathrm{~mL})(p=0,376)$. Del mismo modo, para los lanzamientos realizados desde la zona de 9 metros, se establecen una evolución positiva en cuento al lanzamiento de 9 metros centro $(9 \mathrm{mC})$, pues ha aumentado su uso medio por partido desde el ciclo 1(C1) al ciclo 8(C8), existiendo una diferencia estadísticamente significativa en su evolución ( $p=0,014)$, lo que sugiere una evolución en las características físicas, antropométricas y técnicas de los jugadores de primera línea (laterales y centrales). Sin embargo, el lanzamiento de 9 metros lateral (9mL), ha experimentado una ligera evolución positiva, sin llegar a establecer una diferencia significativa ( $p=0,906)$, ya que a pesar de la mejora de las características, físicas, antropométricas y técnicas mencionadas anteriormente, se añaden dos factores que condicionan el lanzamiento lateral. Siendo por un lado las acciones defensivas pares, y por otro, la perdida de verticalidad. Dificultando de este momo aún más si cabe, este tipo de lanzamientos, así como su evolución en cuanto a su uso y utilización.

Por otro lado, los lanzamientos realizados desde la zona de 7 metros $(7 \mathrm{~m})$ han marcado una regularidad a lo largo de los diferentes ciclos olímpicos estudiados ( $p=0,925)$, lo cual se puede atribuir al incremento paralelo en cuanto a la mejora físico-técnica de los lanzadores además del acceso a las tendencias de parada derivaba del estudio a los porteros. Por el, contrario, los lanzamientos realizados en un contraataque, han aumentado de forma positiva su número de acciones, aunque no se establecen diferencias significativas para este tipo de lanzamiento ( $p=0,213$ ), ya que el contraataque supone una situación, en la mayoría de las ocasiones, ventajosa para el lanzador, al encontrarse con la única oposición del portero, lo cual facilita la consecución del punto (Figura $1)$.

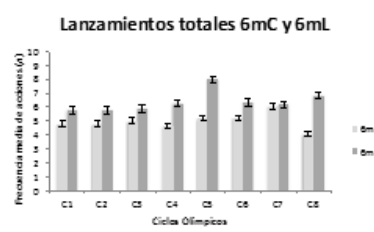

Lanzamientos totales de Contra

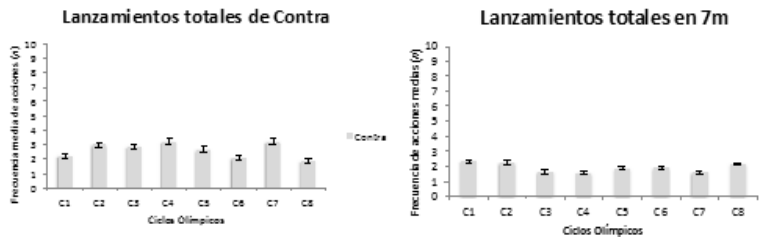

Figura 1: Evolución del total de lanzamientos medios realizados por zonas de lanzamiento y ciclos olímpicos en los que se produjeron.

\section{Lanzamientos con finalización en gol}

Del mismo modo que ha sido realizado el análisis de los lanzamientos totales para conocer la evolución en las tendencias de ataque, es importante conocer el éxito de esos lanzamientos, por tanto a continuación, se presentan los valores de lanzamientos medios por partido que finalizaron en gol desde las zonas analizadas y agrupados por ciclos olímpicos (Tabla 4).

En cuanto al desarrollo y evolución de los lanzamientos finalizados en gol a lo largo de los diferentes ciclos olímpicos en función de la zona de lanzamiento, el análisis de los datos muestra como para la zona de lanzamiento de $6 \mathrm{~m}$, todos los ciclos olímpicos, se marcaron más goles de 6 metros lateral (6mL) que de 6 metros centro (6mC), a excepción del
Tabla 4:

Frecuencia media de acciones de lanzamiento acabadas en gol

\begin{tabular}{|c|c|c|c|c|c|c|c|c|}
\hline & & $\begin{array}{l}\text { Media } \\
\text { (M) }\end{array}$ & $\begin{array}{l}\text { Desviación } \\
\text { (SD) }\end{array}$ & $\begin{array}{l}\text { Mediana } \\
\text { (Me) }\end{array}$ & & $\begin{array}{l}\text { Media } \\
(\mathrm{M})\end{array}$ & $\begin{array}{l}\text { Desviación } \\
\text { (SD) }\end{array}$ & $\begin{array}{l}\text { Mediana } \\
\text { (M) }\end{array}$ \\
\hline \multirow[t]{9}{*}{$6 \mathrm{mC}$} & \multicolumn{8}{|c|}{$9 \mathrm{mC}$} \\
\hline & Ciclo 1 & 4,75 & 3,77 & 4 & Ciclo 1 & 4 & 1,41 & 4,5 \\
\hline & Ciclo 2 & 6 & 6,05 & 3,5 & Ciclo 2 & 3,75 & 1,5 & 4 \\
\hline & Ciclo 3 & 5,75 & 1,7 & 5,5 & Ciclo 3 & 2,75 & 0,95 & 2,5 \\
\hline & Ciclo 4 & 6 & 2,82 & 5,5 & Ciclo 4 & 2,3 & 1,41 & 2 \\
\hline & Ciclo 5 & 6,8 & 2,48 & 6,5 & Ciclo 5 & 2,5 & 1,78 & 2 \\
\hline & Ciclo 6 & 6,6 & 3,92 & 6 & Ciclo 6 & 4,1 & 2,33 & 3,5 \\
\hline & Ciclo 7 & 8 & 3,01 & 7 & Ciclo 7 & 4,7 & 2,11 & 5,5 \\
\hline & Ciclo 8 & 5,2 & 2,39 & 4,5 & Ciclo 8 & 4,7 & 2,71 & 4 \\
\hline $6 \mathrm{~mL}$ & \multicolumn{8}{|c|}{$9 \mathrm{~mL}$} \\
\hline & Ciclo 1 & 7,5 & 4,12 & 7 & Ciclo 1 & 0,5 & 0,57 & 0,5 \\
\hline & Ciclo 2 & 7,5 & 1,91 & 8 & Ciclo 2 & 1 & 1,41 & 0,5 \\
\hline & Ciclo 3 & 7,5 & 3,1 & 8,5 & Ciclo 3 & 0,75 & 0,95 & 0,5 \\
\hline & Ciclo 4 & 6,3 & 3,4 & 6 & Ciclo 4 & 1,3 & 1,16 & 1 \\
\hline & Ciclo 5 & 9,6 & 2,31 & 10 & Ciclo 5 & 1,1 & 0,56 & 1 \\
\hline & Ciclo 6 & 7,6 & 2,45 & 7,5 & Ciclo 6 & 0,8 & 1,03 & 0 \\
\hline & Ciclo 7 & 7,1 & 2,18 & 7 & Ciclo 7 & 0,9 & 0,99 & 1 \\
\hline & Ciclo 8 & 7,3 & 3,56 & 6 & Ciclo 8 & 0,8 & 0,91 & 0,5 \\
\hline \multirow[t]{9}{*}{ Contra } & \multicolumn{8}{|c|}{$7 \mathrm{~m}$} \\
\hline & Ciclo 1 & 2,5 & 2,08 & 2,5 & Ciclo 1 & 2,5 & 2,51 & 2 \\
\hline & Ciclo 2 & 3,5 & 1 & 4 & Ciclo 2 & 3 & 2,44 & 3 \\
\hline & Ciclo 3 & 3 & 1,41 & 2,5 & Ciclo 3 & 2 & 2,3 & 2 \\
\hline & Ciclo 4 & 4,6 & 2,5 & 4,5 & Ciclo 4 & 1,9 & 1,72 & 2 \\
\hline & Ciclo 5 & 3,8 & 1,39 & 3,5 & Ciclo 5 & 2,5 & 1,26 & 2,5 \\
\hline & Ciclo 6 & 2,3 & 1,56 & 2,5 & Ciclo 6 & 2,8 & 2,04 & 3 \\
\hline & Ciclo 7 & 4,4 & 2,22 & 4,5 & Ciclo 7 & 2,3 & 1,49 & 2 \\
\hline & Ciclo 8 & 2,7 & 1,88 & 2 & Ciclo 8 & 3,3 & 1,94 & 2,5 \\
\hline
\end{tabular}

Nota: 6mC: Lanzamientos de 6 metros centro; $6 \mathrm{~mL}$ : Lanzamientos de 6 metros lateral; $9 \mathrm{mC}$ Lanzamientos de 9 metros centro; 9mL: Lanzamientos de 9 metros lateral; Contra: Lanzamientos producidos en un contraataque; $7 \mathrm{~m}$ : Lanzamientos realizados desde los 7 metros.

ciclo 7 (C7). Cabe destacar el hecho que el número de lanzamientos exitoso desde 6 metros centro (6mC) ha ido aumentando positivamente aunque no se establecen diferencias significativas ( $p=0,335)$. Del mismo modo, el lanzamiento de 6 metros lateral $(6 \mathrm{~mL})$ ha mantenido una regularidad temporal, de modo que esta zona de lanzamiento, tampoco registra diferencias estadísticas ( $p=0,297)$. Este hecho obedece a la reforma reglamentaria recogida en el art.8, bajo el concepto de «sanción progresiva» que deriva en un mayor respeto hacia los pivotes por parte de los defensores, lo cual se ha traducido en un mayor número de goles obtenidos desde esta zona de lanzamiento.

Del mismo modo, y por lo que respecta a los lanzamientos de 9 metros, se observa una variación en los lanzamientos exitosos desde esta distancia. Concretamente, desde los 9 metros centro (9mC), se produce una evolución positiva desde el ciclo 5(C5) que va en aumento hasta el ciclo 8 (C8), de modo que la consecución de los lanzamientos desde esta distancia está evolucionando positivamente a pesar de no existir diferencias estadísticas, pero si una tendencia $(p=0,070)$. Sin embargo, la distancia de 9 metros lateral $(9 \mathrm{~mL})$ no ha experimentado una ligera evolución positiva, no mostrando diferencias estadísticas entre los diferentes ciclos $(p=0,855)$, lo cual refuerza la idea presentada en el apartado anterior, donde la evolución de los jugadores en cuanto a mejores cualidades físicas, antropométricas y técnicas, les ha llevado un intentar más lanzamientos desde esta distancia y por consiguiente, obtener un mayor número de goles desde los $9 \mathrm{~m}$.

Por otro lado, en cuanto a la frecuencia de los lanzamientos finalizados en gol tras una acción de contraataque, se observa una evolución irregular aunque de forma general positiva, a pesar de no establecerse

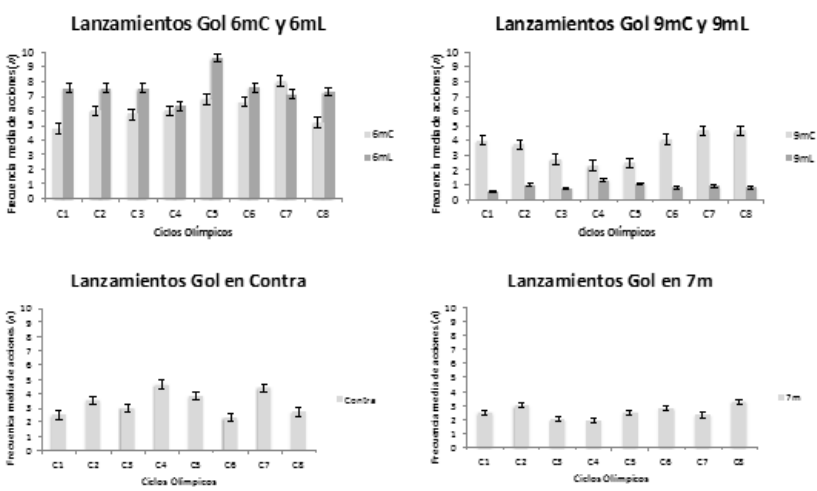

Figura 2: Evolución del total de lanzamientos medios realizados por zonas de lanzamiento y ciclos olímpicos en los que se produjeron 
diferencias significativas $(p=0,146)$, puesto que las paradas realizadas por los porteros en este tipo de acciones se basan en movimientos explosivos que interceptan de forma irregular los lanzamientos efectuados como una consecuencia aleatoria más que como el resultado una correcta ejecución técnica de parada. Finalmente, en cuento a los lanzamientos finalizados en gol desde los 7 metros, al igual que desde otras zonas de lanzamiento, se establece una regularidad que presenta, de formageneral una evolución positiva sin diferencias estadísticas $(p=0,836)$ (Figura 2), posiblemente derivado de la frecuencia de lanzamiento a la mejora de los lanzadores y del estudio de las tendencias de paradas a los porteos.

\section{Efectividades de lanzamiento}

Por último, se establecen las efectividades para cada una de las zonas de lanzamiento. Concretamente, para la zona de 6 metros se produce una evolución positiva en el cómputo general, pero cabe destacar el descenso de la efectividad desde esa zona desde el ciclo 5 (C5) (62,4\%) al ciclo 8 (C8) (57,6\%). Destacando como se ha producido un descenso en la efectividad del lanzamiento de zona de 6 metros centro (6mC) pasando de un 34,1\% de efectividad en el ciclo 1(C1) a un 29,6\% de efectividad en el ciclo 8(C8). Sin embargo este hecho no se coincide con la evolución en las efectividades de lanzamiento de 6 metros lateral (6mL). Desde esta última zona de lanzamiento, se ha experimentado un aumento de la efectividad de lanzamiento, pasando de un $21,7 \%$ en el ciclo 1 (C1) a un 35,3\% de efectividad para el ciclo 8(C8). Este hecho queda justificado como consecuencia de una mejor cobertura defensiva en las zonas centrales, lo cual abre, a partir de las basculaciones de la defensa, más opciones por las zonas laterales, que junto al enriquecimiento de los lanzamientos, ha llevado a un aumento de la efectividad por esas zonas que antes no se daba.

Del mismo modo, la efectividad general desde los 9 metros, ha experimentado un decrecimiento desde el ciclo 3(C3) $(61,4 \%)$ hasta el ciclo 8 (C8) (45,5\%). Concretamente la efectividad desde 9 metros centro (9mC) ha experimentado una variabilidad en su evolución aumentando ligeramente desde el ciclo 1 (C1) con una efectividad del 31\% al ciclo 8 (C8) con una efectividad del 34,1\%. No estableciéndose una tendencia clara en la evolución de la efectividad de 9 metros lateral (9mL), que ha sufrido variaciones de más de 20 puntos en su porcentaje de efectividad. Todo ello sugiere una mejora de las prestaciones defensivas así como de la utilización de sistemas defensivos más abiertos como el 5:1, 3:2:1, el 4:2, el 3:3 y el marcaje al hombre.

En cuanto a las efectividades derivadas de las acciones de contraataque, se experimenta una evolución positiva pero irregular, pasando del 55,6\% en el ciclo 1 (C1) al 71,1\% en el ciclo 8 (C8). Este hecho hace considerar que las efectividades de contraataque registradas responden a una progresiva cualificación de los jugadores de campo, que han mejorado su respuesta técnica y de ejecución ante estas situaciones especiales de juego.

Finalmente, la efectividad de lanzamiento desde los 7 metros (7m) ha experimentado un aumento positivo constante a lo largo de los ciclos

\begin{tabular}{|c|c|c|c|c|c|c|c|}
\hline & \multicolumn{4}{|c|}{ General (\%) Centro (\%) Lateral (\%) } & \multicolumn{3}{|c|}{ General (\%) Centro (\%) Lateral (\%) } \\
\hline $6 \mathrm{~m}$ & & & & & & & \\
\hline Ciclo 1 & 56,3 & 34,1 & 21,7 & Ciclo 1 & 42,9 & 31 & 50 \\
\hline Ciclo 2 & 64,3 & 23,7 & 26,1 & Ciclo 2 & 42,4 & 36 & 12,5 \\
\hline Ciclo 3 & 60,9 & 32,5 & 27,7 & Ciclo 3 & 61,4 & 42,9 & 33,3 \\
\hline Ciclo 4 & 56,7 & 25 & 39,2 & Ciclo 4 & 50,8 & 41,9 & 31,8 \\
\hline Ciclo 5 & 62,4 & 24,3 & 30,6 & Ciclo 5 & 51,5 & 42 & 50 \\
\hline Ciclo 6 & 61,2 & 27,9 & 32,8 & Ciclo 6 & 47,3 & 31,9 & 38,1 \\
\hline Ciclo 7 & 61,9 & 26,4 & 33,3 & Ciclo 7 & 48,3 & 38,1 & 31,6 \\
\hline Ciclo 8 & 57,6 & 29,6 & 35,3 & Ciclo 8 & 45,5 & 34,3 & 33,3 \\
\hline Contra & & & & & & & \\
\hline Ciclo 1 & 55,6 & - & - & Ciclo 1 & 52,6 & - & - \\
\hline Ciclo 2 & 58,3 & - & - & Ciclo 2 & 66,7 & - & - \\
\hline Ciclo 3 & 52,2 & - & - & Ciclo 3 & 61,5 & - & - \\
\hline Ciclo 4 & 71,9 & - & - & Ciclo 4 & 61,3 & - & - \\
\hline Ciclo 5 & 71,7 & - & - & Ciclo 5 & 67,6 & - & - \\
\hline Ciclo 6 & 54,8 & - & - & Ciclo 6 & 73,7 & - & - \\
\hline Ciclo 7 & 68,8 & - & - & Ciclo 7 & 71,9 & - & - \\
\hline Ciclo 8 & 71,1 & - & - & Ciclo 8 & 76,7 & - & - \\
\hline
\end{tabular}

zona de 9 metros; Contra: Lanzamientos realizados en acción de contraataque; 7m: Lanzamientos realizados desde la zona de 7 metros. a excepción del ciclo 3 y 4 que presentó una bajada de 0,2 puntos en la efectividad de ataque. A pesar de ello, muestra la mayor mejora de efectividad, pasando de un 52,6\% en el ciclo 1 (C1) a un 76,7\% de efectividad en el ciclo 8(C8), lo cual denota una mejora en la técnica de los lanzadores que ha ido aumentando con el paso de los años, siendo algunos de ellos, especialistas en este tipo de lanzamientos (Tabla 5).

\section{Discusión}

El lanzamiento a portería es una de las habilidades vitales en balonmano y un claro factor de rendimiento en dicho deporte(Granados et al., 2007). Para que un lanzamiento sea efectivo no solamente es necesario que se realice a la máxima velocidad posible sino que tiene que ir acompañado de una buena precisión (Van den Tillaar, 2003), pues cuanto mayor sea la velocidad de lanzamiento a portería menos tiempo tendrán los defensores y portero para interceptar dicho lanzamiento.

En el presente estudio se han analizado solamente las finales de los distintos campeonatos, lo cual puede suponer una limitación ya que los equipos que disputan los partidos finales lo hacen en un estado de fatiga elevado y la efectividad de los lanzamientos puede verse afectada (Zapartidis et al., 2007). Por otro lado, al tratarse de un estudio histórico de los últimos 30 años, la evolución que ha experimentado la preparación de los jugadores tratando de adaptarse a los nuevos requerimientos del juego es grande (Michalsik, Aagaard, \& Madsen, 2013), pudiéndose verse atenuada en parte, esta posible fatiga acumulada. A pesar de ello, en jugadores expertos y con buena preparación física la fatiga no tiene apenas influencia en la velocidad de lanzamiento aunque sí afectaría a la precisión del mismo (Manchado et al., 2013).

En los últimos 30 años han aumentado los lanzamientos desde $9 \mathrm{~m}$ y $6 \mathrm{~m}$ pero con una efectividad variable debido tanto a la mejor preparación de los porteros como al scouting, elemento este utilizado por los porteros de élite analizando en imágenes los lanzamientos de los rivales en partidos anteriores (Espina-Agulló et al., 2016). La información aportada a los porteros es muy útil ya que la tendencia de todos los jugadores, es tener determinada tendencia a intentar colocar el balón casi siempre los mismos ángulos, pudiendo ser bajo largo, medio largo, alto largo, bajo corto, medio corto, alto corto, así como lanzamiento de recurso, es decir, en trayectoria parabólica, liftada de rosca.

En este estudio se muestra un incremento significativo del número de lanzamientos realizados desde la posición de 9mC, respondiendo probablemente a una evolución en las características físicas, antropométricas y técnicas de los jugadores de primera línea (laterales y centrales) y a una mayor especialización de los primeras líneas de los equipos, que encuentran así mayores facilidades de lanzamiento. Por otro lado, se observa que no se produce una mejora en la eficacia del lanzamiento desde esta posición. Este hecho se podría explicar por la mejora de la eficacia en las acciones del portero y a la oposición ejercida por el equipo defensor (Rivilla-Garcia et al., 2011).

En cuanto a los lanzamientos de $7 \mathrm{~m}$, el número no varía pero se observa una tendencia a la mejora en cuanto a eficacia se refiere. Esta circunstancia se puede atribuir al incremento paralelo en cuanto a la mejora físico-técnica de los lanzadores además del acceso a las tendencias de parada derivada del estudio a los porteros. Además, también podría deberse a la mejora de la técnica de los lanzamientos de $7 \mathrm{~m}$ al incrementarse los llamados especialistas en dicha función, así como por el poco tiempo de reacción del que disponen los porteros (Helm, Reiser, \& Munzert, 2016)

Con respecto a los lanzamientos de $6 \mathrm{~m}$ se observa un ligero incremento en el número de lanzamientos desde las distintas posiciones que podría relacionarse con el aumento general del número de ataques por partido. En la zona central, estas finalizaciones tienen como protagonistas a los pivotes, siendo esta una opción recurrente en lanzamientos por esta zona. En las zonas laterales este aumento sugiere una mejora técnica y física de los lanzadores (Nikolaidis \& Ingebrigtsen, 2013), que han resuelto la falta de verticalidad, con lanzamientos de rosca o liftados que no eran utilizados hace 30 años.

Por último, el número de lanzamientos en contraataque también 
aumenta de forma paralela al número total de lanzamientos durante el partido, ya que el contraataque supone una situación, en la mayoría de las ocasiones, ventajosa para el lanzador, al encontrarse con la única oposición del portero, lo cual facilita la consecución del punto (EspinaAgulló et al., 2016).

Por último, desde el punto de vista de la evolución de los aspectos reglamentarios y por tanto de la evolución del propio juego, encontramos dos elementos que hasta podríamos considerar sesgos del estudio. Por un lado la introducción en los últimos doce años del concepto de saque de centro ejecutado a la máxima velocidad y sin necesidad de que los defensores estén en su propio campo. Por otro, la evolución del concepto reglamentario, y por tanto táctico, del juego pasivo ya que la aplicación de éste ha resultado cada vez más rigurosa hasta nuestros días, siendo dos factores condicionales de los resultados obtenidos sobre la evolución del juego y que deben tenerse en cuenta y ser estudiados en profundidad futuras investigaciones.

\section{Conclusiones}

Es este trabajo, se establece una evolución de los lanzamientos en balonmano desde diferentes zonas de lanzamiento. En primer lugar, se experimenta un aumento del total de lanzamientos debido a un aumento en el número total de ataques. De las diferentes zonas de lanzamiento estudiadas, la zona de 9 metros centro $(9 \mathrm{mC})$, registra un aumento mayor que en el resto de zonas, aunque no ha aumentado su efectividad de forma significativa. En esta línea, se observa una evolución dispar en la efectividad de 6 metros. Concretamente, 6 metros lateral $(6 \mathrm{~mL})$, a diferencia de 6 metros centro $(6 \mathrm{mC})$ se produce un aumento de la efectividad como consecuencia de la mejora técnica de los pivotes, los cuales realizan lanzamientos más variados. Para el resto de lanzamientos, no se observa una evolución en la eficacia de forma significativa.

\section{Referencias}

Debanne, T., \& Laffaye, G (2011). Predicting the throwing velocity of the ball in handball with anthropometric variables and isotonic tests. Journal of Sports Sciences, 29(7), 705-13.

Espina-Agullo, J. J. (2013). Historical, tactical and structural analysis of the 4:2 defensive play system in handball. Journal of Human Sport and Exercise, 8(3), $2-5$.

Espina-Agulló, J. J., \& Jove-Tossi, M.A.(2012). Historical and tactical development of the 6:0 defence system in handball. Journal of Human Sport and Exercise, 7(Special Issue.2), 454-467.

Espina-Agulló, J. J., Pérez-Turpin, J. A., Jiménez-Olmedo, J. M., Penichet-Tomás, A., \& Pueo, B. (2016). Effectiveness of male handball goalkeepers: Ahistorical overview 1982-2012. Journal of Performance Analysis in Sport, 1(16), 143156.

García, J. A., Moreno, F. J., Reina, R., \& Menayo, R. (2011). La velocidad y la precisión en el lanzamiento en jóvenes jugadores de balonmano en función de la concentración de la práctica. Retos. Nuevas Tendencias en Educación Física, Deportey Recreación, (19), 43-46.

González Fimbres, R. A., Griego Amaya, H., Cuevas Castro, C. S., \& Hernández Cruz, G. (2016). Influence of training load volume and intensity on heart rate recovery. Retos: Nuevas Tendencias En Educación Física, Deportey Recreación, (30), 180-183.

GonzalezHernandez, J., \& Valadez Jimenez,A.(2016). Personality and psychological response in athletes. Temporal and adaptive representation of the person-sport process. Retos. Nuevas Tendencias En Educacion Física, Deporte y Recreación, (30), 211-215.

Granados, C., Izquierdo, M., Ibañez, J., Bonnabau, H., \& Gorostiaga, E. M. (2007) Differences in physical fitness and throwing velocity among elite and amateur female handball players. International Journal of Sports Medicine, 28(10), 860-867.

Helm, F., Reiser, M., \& Munzert, J. (2016). Domain-specific and unspecific reaction times in experienced team handball goalkeepers and novices. Frontiers in Psychology, 7, 882-893.

Hughes, M. (2004). Notational analysis - Amathematical perspective. International Journal of Performance Analysis in Sport, 4(2), 97-139.

James, N., Taylor, J., \& Stanley, S. (2007). Reliability procedures for categorical data in PerformanceAnalysis. International Journal of Performance Analysis in Sport, 7(1), 1-11.

Jimenez-Olmedo, J. M., Pueo, B., \& Penichet-Tomás, A. (2016). Defensive systems during the Men's European University Beach Volleyball Championship. Journal of Physical Education and Sport, 16(3), 945-950.

Jimenez-Olmedo, J. M., Pueo, B., Penichet-Tomás, A., Chinchilla-Mira, J. J., \& Perez-Turpin, J.A. (2017). Physiological work areas in professional beach volleyball: A case study. Retos. Nuevas Tendencias en Educación Fisica, Deporte y Recreación, (31), 94-97.

Manchado, C., Tortosa-Martínez, J., Vila, H., Ferragut, C., \& Platen, P. (2013). Performance Factors in Women $1 \frac{1}{4}$ S Team Handball. Physical and physiological aspects - A review. Journal of Strength and Conditioning Research, 27(6), 1708-1719.

Marques, M. C., van den Tilaar, R., Vescovi, J. D., \& Gonzalez-Badillo, J. J. (2007). Relationship between throwing velocity, muscle power, and barvelocity during bench press in elite handball players. International Journal of Sports Physiology and Performance, 2(4), 414-422.

Michalsik, L. B., Aagaard, P., \& Madsen, K. (2013). Locomotion characteristics and match-induced impairments in physical performance in male elite team handball players. International Journal of Sports Medicine, 34(7), 590-9.

Murillo Lorente, V., Alvarez Medina, J., \& Manomelles Marqueta, P. (2016). Control of training loads through perceived exertion. Prediction of heart rate. Retos. Nuevas Tendencias En Educacion Física, Deporte y Recreación, 30, 82-86.

Nikolaidis, P. T., \& Ingebrigtsen, J. (2013). Physical and physiological characteristics of elite male handball players from teams with a different ranking. Journal of Human Kinetics, 38, 115-24.

Pascual, N., Alzamora, E. N., Martínez Carbonell, J. A., \& Pérez Turpin, J. A. (2015).Analysis of different teaching methods in young soccer players. Retos. Nuevas Tendencias En Educacion Física, Deporte y Recreación, (28), 94-97.

Peñaloza Gómez, R., Jaenes Sanchez, J. C., Mendez-Sanchez, M. del P., \& JaenesAmarillo, P. (2016). The explanatory power of anxiety in the mood of Spanish athletes. Retos. Nuevas Tendencias En Educacion Física, Deporte y Recreación, (30), 207-210.

Reynoso-Sanchez, L.-F., Hernandez-Cruz, G., Lopez-Walle, J., Rangel-Colmenero, B., Quezada-Chacon, J.-T., \& Jaenes Sanchez, J. C. (2016). Recovery-stress balance throughout a season in volleyball university players. Retos. Nuevas Tendencias En Educacion Física, Deportey Recreación,(30), 193-197.

Rivilla-Garcia, J., Grande, I., Sampedro, J., \& Van den Tillaar, R. (2011). Influence of opposition on ball velocity in the handball jump throw. Journal of Sports Science \& Medicine, 10(3), 534-539.

Rogulj, N., Srhoj, V., Nazor, M., Srhoj, L., \& Cavala, M. (2005). Some anthropologic characteristics of elite female handball players at different playing positions. Collegium Antropologicum, 29(2), 705-709.

Van den Tillaar, R. (2003). Influence of instruction on velocity and accuracy of overarm throwing. Perceptual and Motor Skills, (96), 731-742.

Van den Tillaar, R. (2004). Effect of Different Training Programs on the Velocity of Overarm Throwing: A brief review. Journal of Strength and Conditioning Research, 18(2), 388-396.

Van den Tillaar, R., \& Ettema, G. (2004a). A force - velocity relationship and coordination patterns in overarm throwing. Journal of Sports Science \& Medicine,3(4), 211-219.

Van den Tillaar, R., \& Ettema, G. (2004b). Effect of body size and gender in overarm throwing performance. European Journal of Applied Physiology, 91(4), 413418.

Van den Tillaar, R., \& Ettema, G. (2009). Acomparison of overarm throwing with the dominant and nondominant arm in experienced team handball players. Perceptual and Motor Skills, 109(1), 315-326.

Vila, H., Manchado, C., Rodriguez, N., Abraldes, J.A., Alcaraz, P. E., \& Ferragut, C. (2012).Anthropometric profile, vertical jump, and throwing velocity in elite female handball players by playing positions. Journal of Strength and Conditioning Research, 26(8), 2146-55.

Wagner, H., Buchecker, M., von Duvillard, S. P., \& Müller, E. (2010). Kinematic description of elite vs. low level players in team-handball jump throw. Journal of Sports Science and Medicine, 9(1), 15-23.

Wagner, H., Buchecker, M., Von Duvillard, S. P., \& Müller, E. (2010). Kinematic comparison of team handball throwing with two different arm positions. International Journal of Sports Physiology and Performance, 5(4), 469-483.

Wagner, H., Pfusterschmied, J., Klous, M., Von Duvillard, S. P., \& Müller, E. (2012). Movement variability and skill level of various throwing techniques. Human Movement Science, 31(1), 78-90.

Zapartidis, I., Gouvali, M., Bayios, I., \& Boudolos, K. (2007). Throwing effectiveness and rotational strength of the shoulder in team handball. Journal of Sports Medicine and Physical Fitness, 47(2), 169-178. 